\title{
Enhancement of cardiac contractility by refractory period stimulation in conjunction with cardiac resynchronization therapy
}

\author{
Farong Shen ${ }^{1 *}$, Yuanwei Liu ${ }^{2}$, Hongfeng Jin ${ }^{2}$, Gong Chen ${ }^{3}$ and Xiaohong Zhou ${ }^{3}$ \\ ${ }^{1}$ Greentown Cardiovascular Disease Hospital, Hangzhou, P.R. China \\ ${ }^{2}$ Zhejiang Hospital, Hangzhou, P.R. China \\ ${ }^{3}$ CRDM China, Medtronic (Shanghai), Inc., Shanghai, P.R. China
}

\begin{abstract}
Background: Cardiac resynchronization therapy (CRT) improves cardiac function in patients with heart failure by synchronizing contraction of the right and left ventricles $(\mathrm{RV}, \mathrm{LV})$. This study was to determine if electrical stimulation during the refractory period of bi-ventricular (BiV) pacing would provide additional LV mechanical improvement.
\end{abstract}

Methods: 16 patients receiving CRT-ICD devices were enrolled. After the CRT leads were in place, the leads were connected to an EP stimulator for acute delivery of electrical stimuli during the absolute refractory period of each cardiac beat for a 30-sec interval. Four types of refractory period stimulation were delivered: stimulation at the local LV pacing site during intrinsic rhythm, at the local RV pacing site during intrinsic rhythm, at the local LV pacing site during BiV pacing, and simultaneously at $\mathrm{LV}$ and $\mathrm{RV}$ during $\mathrm{BiV}$ pacing. The $\mathrm{LV}$ pressure was recorded for $\mathrm{dp} / \mathrm{d}$ tmax measurement to assess changes of cardiac contractility.

Results: Overall LV dp/dtmax increased to $1453 \pm 522 \mathrm{mmHg} / \mathrm{Sec}$ during refractory period stimulation from a baseline of $1383 \pm 476 \mathrm{mmHg} / \mathrm{Sec}(\mathrm{P}=0.013)$. The $\mathrm{LV} \mathrm{dp} / \mathrm{dtmax}$ was significantly increased by an average $12.8 \%$ when refractory period stimulation was simultaneously delivered at the LV free wall and the RV apex during $\mathrm{BiV}$ pacing. There was no significant improvement in $\mathrm{LV}$ dp/dtmax by other three types of stimulation.

Conclusion: Refractory period stimulation, when delivered to both RV and LV simultaneously, can improve LV mechanical function on top of BiV pacing, which may provide additional benefits for CRT patients.

\section{Introduction}

Congestive heart failure (CHF) is a syndrome caused by impaired ventricular function. Despite improvements in pharmacological treatment, the prognosis for patients with heart failure remains poor the risk of death annually is $5-10 \%$ in patients with mild symptoms and $30-40 \%$ in those with advanced disease [1,2]. More recently, cardiac resynchronization therapy (CRT) that synchronizes cardiac contraction of left and right ventricles (LV, RV) has been shown to provide further morbidity and mortality benefits in addition to pharmacologic therapy in patients with ventricular dyssynchrony (wide QRS) and a reduced left ventricular ejection fraction $\leq 35 \%[3,4]$. However, approximately $30 \%$ of patients who receive CRT are non-responders [5-7].

Electrical stimulation during refractory period is a newly developed method to enhance myocardial contractility, which requires beat-tobeat electrical stimulation during the ventricular absolute refractory period (ARP) [8-12]. Research in animals demonstrated beneficial effects of refractory period stimulation on cardiac function in a canine heart failure model [13-15]. Studies in humans have examined the safety and efficacy of cardiac contractility modulation by refractory period stimulation in patients who have CHF with narrow QRS width [16-20]. In the study by Borggrefe et al. patients with heart failure experienced better exercise tolerance and quality of life with active cardiac contractility modulation therapy compared to without [16].
Today's stimulation system of cardiac contractility modulation used in patients with CHF utilizes two right ventricular leads that attach to the septum of the right ventricle and apply electrical stimulation during the absolute refractory period $[16,18]$. The localized improvement, however, might not be synchronized with global activity of ventricles, especially in patients with impaired synchronization of activation conduction in ventricles. Thus, the purpose of the present study was to test the hypothesis that the magnitude of the myocardial contractility improvement by refractory period stimulation would be greater if the stimuli were applied through the CRT lead system.

\section{Methods}

The present study was a single-center, non-randomized acute study in CRT-indicated patients who underwent the implantation of a CRTICD. The study protocol was approved by the hospital Investigational

Correspondence to: Farong Shen, Department of Cardiology, Greentown Cardiovascular Disease Hospital, 409, Gudun Road, Xihu district, Hangzhou, P.R. China, E-mail: shenfarong2011@163.com

Key words: cardiac resynchronization therapy, refractory period stimulation, cardiac contractility, heart failure

Received: June 26, 2015; Accepted: July 20, 2015; Published: July 24, 2015 
Research Board and in compliance with the Declaration of Helsinki and with the laws and regulations in China. All patients completed written informed consent.

\section{Patient selection}

The study enrolled sixteen patients between October 2011 and December 2012. Enrolled patients met the following criteria: (1) patients were indicated for receiving CRT-ICD, (2) patients underwent the placement of intracardiac leads for CRT-ICD, (3) patients were greater than 18 years of age, and (4) patients were willing and able to give informed consent.

Patients were excluded if (1) patients had experienced angina in the last three months, (2) patients' measurement of left ventricular (LV) pressure was not technically feasible (e.g. atrial fibrillation, frequent premature ventricular contractions), (3) patients were enrolled in other clinical studies that would confound the results of this study, or (4) patients were pregnant. Demographic and medical history was collected at enrollment.

\section{Study procedures}

Lead placement: Each patient underwent standard procedures of CRT-ICD implantation under local anesthesia. Briefly, the atrial lead (model 4574, Medtronic, Inc., Minnesota, USA) was placed in the right atrial appendage, the RV lead (Model 5076, Medtronic, Inc., Minnesota, USA) was placed in the RV with the tip attaching to the apex for RV apical pacing, and the LV lead (Models 4193, 4194, or 4195, Medtronic, Inc., Minnesota, USA) was placed in a tributary of the coronary sinus for stimulating the LV free wall. The LV lead was targeted to a lateral or a postero-lateral cardiac vein. The procedure of lead placement was performed under fluoroscopy. After all leads were positioned, the leads were connected to an EP stimulator (Model Transtar MX9505T, MedLab-U, Nanjing Medease Science and Technology Co., Ltd, Nanjing, China) for delivering refractory period stimulation as described below. Once the tests were completed, all leads were connected to a CRT-ICD device that was then implanted inferior to the left clavicle.

Hemodynamic monitoring: An LV pressure catheter (Model Transtar MX9505T, Smith Medical ASD, Dublin, OH 43016 USA) was inserted via the left radial artery into the LV chamber. The pressure transducer was then connected with a data acquisition system (Model Transtar MX9505T, MedLab-U, Nanjing Medease Science and Technology co., Ltd, Nanjing, China) that recorded the LV pressure at a sampling rate of $1 \mathrm{KHz}$ along with simultaneous ECG. Pressure data were analyzed offline by a custom Matlab program. The LV pressure parameters for each beat consisted of LV systolic pressure, LV end-diastolic pressure, $\mathrm{dp} / \mathrm{dt}_{\max }$, and heart rate. Pressure parameters measured during premature cardiac beats were excluded in the analysis.

Test of refractory period stimulation: The absolute refractory period (ARP) of ventricles was measured in each patient to ensure no refractory period stimuli would be delivered during the later phase of the ARP. Ten S1 stimuli were delivered with the amplitude at twice pacing threshold and S1-S1 interval of $90 \%$ of the intrinsic R-R intervals based on the ECG or 10 beats faster than the sinus rate. The last $\mathrm{S} 1$ was followed by an S2 with S1-S2 starting at approximately $300 \mathrm{msec}$ at 10 msec step-down until the S2 could not capture the ventricle. The ARP was then recorded. Once the ARP was determined and all S1 stimuli could capture the heart, the refractory period stimulation was tested at the early phase of the ARP to avoid any potential arrhythmias.
Refractory period stimulation was delivered on every beat after a $30 \mathrm{msec}$ delay following either an intrinsic QRS complex during sinus rhythm or bi-ventricular (BiV) pacing. The EP device was designed to sense the local intrinsic activation so that the stimuli could be delivered at the specified time after the local signal sensing. The BiV pacing was achieved by delivering S1 pacing through the tip electrode of the LV lead and the tip electrode of the RV lead at a rate $10 \%$ faster than the intrinsic rate to continuously capture ventricles. Refractory period stimulation for each cardiac beat consisted of 3 monophasic pulses, each separated by $10 \mathrm{msec}$ and having amplitude of 8 volts. Four types of refractory period stimulation were tested. Test 1: refractory period stimulation was locally delivered via the tip electrode of the LV lead and the RV coil (return electrode) during intrinsic sinus rhythm. Test 2: stimulation was locally delivered via the tip electrode of the RV lead at the RV apex and the RV coil during intrinsic sinus rhythm. Test 3: stimulation was locally delivered via the tip electrode of the LV lead and the RV coil during $\mathrm{BiV}$ pacing. Test 4: stimulation was simultaneously delivered at the LV tip electrode and the RV tip electrode during BiV pacing and the return electrode was the RV coil electrode. Each test was performed for $30 \mathrm{sec}$ with a 2 -minute recovery interval between adjacent tests.

\section{Data analysis}

The baseline data was defined as the 30 -sec data that was collected before corresponding test, e.g., the 30-sec data of the intrinsic rhythm before tests 1 and 2 and the 30 -sec data of $\mathrm{BiV}$ pacing before tests 3 and test 4 . The test data was defined as the data in which refractory period stimulation was delivered. The beat-to-beat LV pressure data was analyzed for each of 30-sec data segments and an averaged value from each 30-sec data segment was then obtained for further comparisons. Absolute values and percent changes (measured variables during test over the baseline) were compared between baseline and during tests described above. Data were presented as mean $\pm \mathrm{SD}$ or percentage wherever applicable. Student paired or unpaired t test for two means was used for comparisons. Statistically significant results were defined as a probability of $\mathrm{p}<0.05$.

\section{Results}

All patients (age: $65 \pm 11$ years old, 11 males) were indicated for CRT-ICD and received optimal medical therapy prior to CRT-ICD implantation. The average left ventricular ejection fraction was $29 \pm$ $6 \%$, QRS duration was $156 \pm 29 \mathrm{msec}$, and the NYHA classification was $3 \pm 0.7$. At the CRT-ICD implantation and tests of refractory period stimulation, all 16 patients were under beta-blocker therapy, 13 (81\%) under angiotensin-converting enzyme inhibitor, and 10 patients $(63 \%)$ under diuretics. Most patients $(14,86 \%)$ had dilated cardiomyopathy and 2 patients had a history of ventricular tachyarrhythmia. There were a total of 55 tests available for the data analysis in 16 patients.

Refractory period stimulation produced a positive effect on cardiac mechanical function as shown in LV pressure recording in Figure 1. When the refractory period stimulation was delivered via both RV and LV electrodes during BiV pacing (Figure 1B), the LV pressure and its $\mathrm{dp} /$ $\mathrm{dt}_{\max }$ were greater than during $\mathrm{BiV}$ pacing only (Figure $1 \mathrm{~A}$ ), suggesting a cardiac mechanical improvement. Overall, $\mathrm{LV} \mathrm{dp} / \mathrm{dt}_{\max }$ increased to $1453 \pm 522 \mathrm{mmHg} / \mathrm{Sec}$ during refractory period stimulation from the baseline $1383 \pm 476 \mathrm{mmHg} / \mathrm{Sec}$ without refractory period stimulation ( $\mathrm{P}=0.013$ vs. stimulation tests), representing an overall increase of $5.2 \pm$ $10.4 \%$ in $\mathrm{LV} \mathrm{dp} / \mathrm{dt}_{\text {max }}$ by refractory period stimulation.

The response to refractory period stimulation varied depending 
A
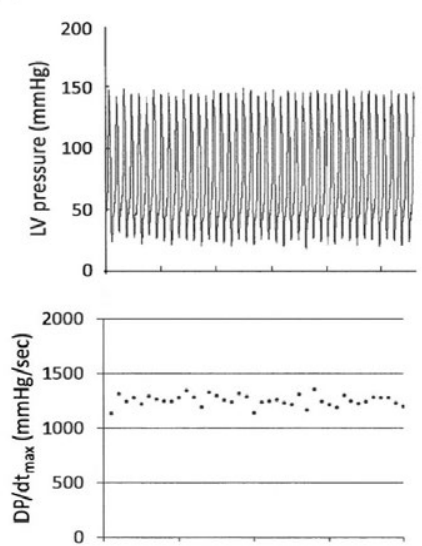

B
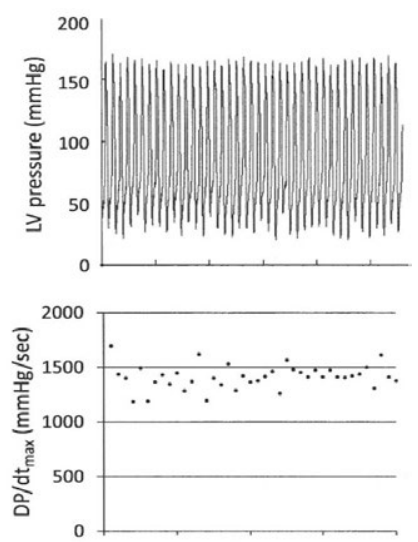

Figure 1. Example of recording of LV pressure with $\mathrm{dp} / \mathrm{dt}$. Panel A shows the 30 -sec $\mathrm{LV}$ pressure recording (upper) and the corresponding measured $\mathrm{dp} / \mathrm{dt}_{\max }$ (bottom) during BiV pacing in one patient. Panel B shows the 30 -sec LV pressure recording (upper) and the corresponding measured $\mathrm{dp} / \mathrm{dt}_{\max }$ (bottom) during BiV pacing plus refractory period stimulation in the same patient.

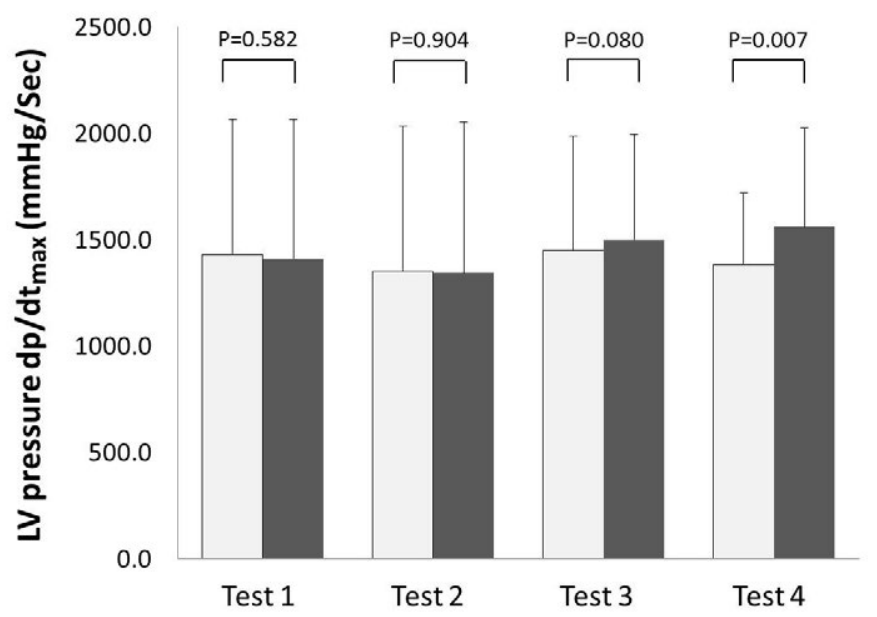

Figure 2. $\mathrm{LV}$ pressure $\mathrm{dp} / \mathrm{dt}_{\max }$ (mean and SD) during baseline (white bars) and four types of refractory period stimulation (black bars). Test 1: refractory period stimulation locally delivered to the LV electrode during sinus rhythm; Test 2: stimulation locally delivered to the RV lead during sinus rhythm; Test 3: stimulation locally delivered to the LV lead during BiV pacing; and Test 4: stimulation simultaneously delivered to the LV lead and RV lead during $\mathrm{BiV}$ pacing.

on how the stimulation was delivered. As shown in Figure 2, there was no significant improvement in $\mathrm{LV} \mathrm{dp} / \mathrm{dt}_{\max }$ by refractory period stimulation that was delivered locally either in the left ventricle or the right ventricle (Tests 1-3). However, when refractory period stimulation was delivered simultaneously at the LV free wall and the $\mathrm{RV}$ apex, the $\mathrm{LV} \mathrm{dp/dt}{ }_{\max }$ was significantly increased by an average of $12.8 \%$ (Test 4 , Figure 2).

The responses to refractory period stimulation in the present study were classified into two categories based on the criteria of

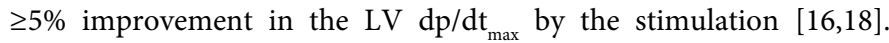
Insignificant response was assigned if the change in $\mathrm{LV} \mathrm{dp} / \mathrm{dt}_{\max }$ was $<5 \%$ during refractory period stimulation compared to the baseline while significant response was assigned if the change in $\mathrm{LV} \mathrm{dp} / \mathrm{dt}_{\max }$ was greater than or equal to $5 \%$ during the stimulation. Of a total of 55 appropriate stimulation tests, 24 (44\%) stimulation tests generated

significant responses and 31 tests (56\%) yielded insignificant responses. The averaged percent change in $\mathrm{LV} \mathrm{dp} / \mathrm{dt}_{\max }$ from the baseline was $14.1 \pm$ $8.8 \%$ for significant responses and $-0.9 \pm 4.7 \%$ for insignificant responses $(\mathrm{P}<0.0001$ between two responses). When the maximum response (one value) was selected from multiple tests of refractory period stimulation in each of 16 patients, the averaged maximum percent change in LV $\mathrm{dp} / \mathrm{dt}_{\max }$ produced by refractory period stimulation was $12.3 \pm 9.1 \%$ over the baseline value. Of 16 patients, $12(75 \%)$ patients experienced at least one significant response out of multiple tests and an averaged maximum percent change in the $\mathrm{LV} \mathrm{dp/dt}$ by refractory period stimulation was $15.8 \pm 7.5 \%$ from the baseline; $4(25 \%)$ patients had only insignificant responses during multiple tests of refractory period stimulation (the averaged maximum percent change in the $\mathrm{LV} \mathrm{dp} / \mathrm{dt}_{\max }$ of $1.8 \pm 3.0 \%$ from the baseline).

Left ventricular systolic pressure during the intrinsic sinus rhythm was $138.0 \pm 11.7 \mathrm{mmHg}$ at the baseline and $136.1 \pm 11.4 \mathrm{mmHg}$ during refractory period stimulation ( $\mathrm{P}=0.103 v$ s. during sinus rhythm). The LV systolic pressure was $127.8 \pm 19.8 \mathrm{mmHg}$ during the baseline $\mathrm{BiV}$ pacing and $132.8 \pm 19.6 \mathrm{mmHg}$ during the $\mathrm{BiV}$ pacing plus refractory period stimulation ( $\mathrm{P}=0.004 v s$. baseline $\mathrm{BiV}$ pacing). The $\mathrm{LV}$ diastolic pressure during the intrinsic sinus rhythm was $15.7 \pm 11.6 \mathrm{mmHg}$ at the baseline and $13.2 \pm 11.3 \mathrm{mmHg}$ during refractory period stimulation ( $\mathrm{P}=0.293$ vs. sinus rhythm without refractory period stimulation). The LV diastolic pressure was $14.1 \pm 8.8 \mathrm{mmHg}$ during baseline BiV pacing and $12.6 \pm 7.6 \mathrm{mmHg}$ during $\mathrm{BiV}$ pacing plus refractory period stimulation $(\mathrm{P}=0.094)$.

\section{Discussion}

\section{Major findings}

This study demonstrated for the first time that refractory period stimuli delivered through the existing CRT lead system could improve

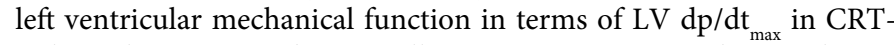
indicated patients. The overall maximum percent change during refractory period stimulation compared to the baseline could reach $12 \%$ improvement in $\mathrm{LV} \mathrm{dp} / \mathrm{dt}_{\max }$. The response to refractory period stimuli was significant when the stimuli were delivered simultaneously to both ventricles during bi-ventricular pacing.

\section{Prior studies}

Studies in both animals and humans have demonstrated the enhancement of ventricular contractile performance by electrical stimuli applied to cardiac tissue during the absolute refractory period [8-19]. In mechanism studies of isolated ventricular muscle, monophasic pulses delivered during the refractory period can modulate not only the cardiac contractility but also the calcium $\left(\mathrm{Ca}^{+2}\right)$ flux across the sarcolemma [8-10]. The administration of beta-blockers in that study cannot diminish the contractility modulation by the electrical stimulation during the cardiac refractory period $[8,11,14]$. The studies in canine heart failure model demonstrated that in addition to the acute and chronic effects of electrical modulation on contractility, changes in gene expression (genetic remodeling) might contribute to a therapeutic effect of electrical modulation on the failing heart [13-15].

Clinical evaluations of electrical cardiac contractility modulation for heart failure therapy were carried out in a cross-over study in 120 patients (Fix-HF4) [16] and a randomized trial in 420 heart failure patients (Fix-FH5) [18]. Both studies used an implantable device (Optimizer) that delivers an approximately $8 \mathrm{~V}$ dual biphasic waveform $40 \mathrm{~ms}$ after a ventricular depolarization through the conventional lead 
configuration with two stimulating electrodes placed in the septum of the right ventricle $[16,18]$. The results from the Fix-HF4 clinical study showed modest improvements in $\mathrm{VO}_{2 \text { peak }}$ and quality of life (MLWHFQ) when electrical cardiac contractility modulation was delivered at regular intervals for a total of 7 hours daily [16]. The results of the Fix-HF5 clinical trial in CHF patients with narrow QRS duration demonstrated that electrical cardiac contractility modulation significantly improved the clinically relevant secondary endpoints of peak ventilatory oxygen uptake (pVO2) and quality of life though there were no significant differences in the primary efficacy endpoint of anaerobic threshold [18]. Another clinical study was performed to compare the effect of refractory period stimulation on the changes in contractility when cardiac contractility modulation stimuli were delivered in the RV compared to the LV in patients with ischemic or idiopathic dilated cardiomyopathy [20]. A single temporary pacing lead was inserted into the RV to stimulate the ventricular septum $(n=6)$, or into a coronary vein to stimulate the LV epicardium $(n=12)$. That study found that both LV and RV stimulation with a single biphasic pulse increased LV pressure $\mathrm{dp} / \mathrm{dt}_{\max }$ to a similar degree (an increase of $9.1 \pm 4.5 \%$ by left ventricular stimulation and $7.1 \pm 0.8 \%$ by right ventricular stimulation) [20].

\section{Present study}

Consistent with the prior investigations [16-19], the present study also demonstrated an overall improvement in LV mechanical function by electrical stimuli during the absolute refractory period of each cardiac beat in patients with heart failure. The uniqueness of the present study was to use the existing CRT lead configurations for delivering refractory period stimulation in CRT-indicated patients. Furthermore, the present study demonstrated that refractory period stimuli that were delivered locally in either the right or left ventricle did not significantly improve the $\mathrm{LV} \mathrm{dp} / \mathrm{dt}_{\max }$. Only when refractory period stimuli were delivered simultaneously to both right and left ventricles, did the stimuli generate a significant improvement in the $\mathrm{LV} \mathrm{dp} / \mathrm{dt}_{\max }$ compared to the baseline value. These findings suggest that locally delivered stimuli in either the right or left ventricle might not synchronize with global function in CRT patients who had dyssynchrony between the right and left ventricles. Another hypothesis was that when refractory period stimuli were delivered simultaneously to both ventricles in the present study, the area affected by stimuli might be increased for a greater response in the $\mathrm{LV} \mathrm{dp/dt} t_{\max }$. Thus, the stimulation delivery method in conjunction with CRT system in the present study played a significant role in achieving contractility enhancement.

An acute increase in $\mathrm{LV} \mathrm{dp/ \textrm {dt } _ { \text { max } }}$ greater than $5 \%$ was used as a criterion for the positive response to an appropriate lead placement in the RV septum and acceptance of device implant for electrical cardiac contractility modulation [16-19]. When this criterion was used to define the response to refractory period stimulation in the present study, $75 \%$ of 16 tested patients met the response criterion. Thus, the non-responder rate to the refractory period stimulation delivered via the CRT lead system could be $25 \%$ based on the present study. It is unknown whether the response can be further improved by modulating the parameters of refractory period stimulation.

\section{Limitations}

It has long been recognized that the $\mathrm{LV} \mathrm{dp} / \mathrm{dt}_{\max }$ represents the contractility of ventricular myocardium. However, the association of the $\mathrm{LV} \mathrm{dp/dt}{ }_{\text {max }}$ with clinical assessments and patient performance is unknown. It is also unclear whether a $5-15 \%$ increase of the $\mathrm{LV} \mathrm{dp} / \mathrm{dt}_{\max }$ would contribute to a significant improvement in clinical performance in heart failure patients.

The present study was an acute investigation without follow up. Thus, it is unknown whether a long-term application of refractory period stimulation through a CRT lead system would lead to any longterm clinical benefits such as improvements in echocardiographic and cardiac functional assessments. Long-term clinical trials are thus needed.

$\mathrm{BiV}$ pacing used in the present study was not equivalent to true CRT pacing due to the limitation of the EP stimulator that did not have a full capability for true CRT pacing,

The present study was performed in 16 patients. This small sample size may limit the interpretation of the findings in the present study to the CRT-indicated population. A large population study will be required to confirm the findings in the present study.

\section{Clinical implications}

CRT restores the synchronization of the left and right ventricles from the dyssynchrony caused by inter/intra-ventricular conduction delay in selected patients (systolic dysfunction and wide ECG QRS duration). However, the CRT non-responder rate remains high, accounting for one third of CRT receivers [5-7]. Cardiac contractility modulation has been shown to improve ventricular contractility, but the current cardiac contractility modulation device only delivers stimuli at the septum of the right ventricle without helping in synchronization. Moreover, today's device of cardiac contractility modulation must be used in patients with implantable cardioverter-defibrillator $[16,18]$, which means the cardiac electrical therapy with two independent implantable devices. There is the possibility that ventricular mechanical function can be further improved if CRT works in conjunction with cardiac contractility modulation [21], e.g., synchronization plus contractility enhancement by one single implantable device. The present study demonstrates for the first time the feasibility that cardiac contractility modulation in terms of refractory period stimulation, when delivered simultaneously to both the left and right ventricles through an existing CRT-ICD lead system, can provide additional improvement of LV dp/ $\mathrm{dt}_{\max }$. This finding may add clinical benefits on top of CRT in CRT-ICD patients, especially for those who are CRT non-responders. Another applicable possibility is to deliver refractory period stimulation only in the event when an acute weakening of myocardial contractility is detected. By doing so, refractory period stimulation is delivered on demand with battery energy saving.

\section{References}

1. (1999) Effect of metoprolol $\mathrm{CR} / \mathrm{XL}$ in chronic heart failure: Metoprolol CR/XL Randomised Intervention Trial in Congestive Heart Failure (MERIT-HF) Lancet 353: 2001-2007. [Crossref]

2. Ahmed A, Perry GJ, Fleg JL, Love TE, Goff DC Jr, et al. (2006) Outcomes in ambulatory chronic systolic and diastolic heart failure: a propensity score analysis. $\mathrm{Am}$ Heart J 152: 956-966. [Crossref]

3. Cleland JG, Daubert JC, Erdmann E, Freemantle N, Gras D, et al. (2005) The effect of cardiac resynchronization on morbidity and mortality in heart failure. $N$ Engl J Med 352: 1539-1549. [Crossref]

4. Bristow MR, Saxon LA, Boehmer J, Krueger S, Kass DA, et al. (2004) Cardiacresynchronization therapy with or without an implantable defibrillator in advanced chronic heart failure. $N$ Engl J Med 350: 2140-2150. [Crossref]

5. Aranda JM, Woo GW, Schofield RS, Handberg EM, Hill JA, et al. (2005) Integrating advanced heart failure treatment with optimal device function management of heart failure after cardiac resynchronization therapy. J Am Coll Cardiol 46: 2193-2198. [Crossref] 
6. Bank AJ, Kelly AS, Burns KV (2006) The role of pacemaker optimization in CRT nonresponders. Circulation 114: II_612-613.

7. Auricchio A, Prinzen FW (2011) Non-responders to cardiac resynchronization therapy: the magnitude of the problem and the issues. Circ J 75: 521-527. [Crossref]

8. Lyon AR, Samara MA, Feldman DS (2013) Cardiac contractility modulation therapy in advanced systolic heart failure. Nat Rev Cardiol 10: 584-598. [Crossref]

9. Mohri S, Shimizu J, Mika Y, Shemer I, Wang J, et al. (2003) Electric currents applied during refractory period enhance contractility and systolic calcium in the ferret heart. Am J Physiol Heart Circ Physiol 284: H1119-H1123. [Crossref]

10. Gupta RC, Mishra S, Rastogi S, Wang M, Rousso B, et al. (2009) Ca(2+)-binding proteins in dogs with heart failure: effects of cardiac contractility modulation electrical signals. Clin Transl Sci 2: 211-215. [Crossref]

11. Winter J, Brack KE, Ng GA (2011) The acute inotropic effects of cardiac contractility modulation $(\mathrm{CCM})$ are associated with action potential duration shortening and mediated by $\beta$ adrenoceptor signalling. J Mol Cell Cardiol 51: 252-262. [Crossref]

12. Brunckhorst CB, Shemer I, Mika Y, Ben-Haim SA, Burkhoff D (2006) Cardiac contractility modulation by non-excitatory currents: studies in isolated cardiac muscle. Eur J Heart Fail 8: 7-15. [Crossref]

13. Rastogi S, Mishra S, Zacà V, Mika Y, Rousso B, et al. (2008) Effects of chronic therapy with cardiac contractility modulation electrical signals on cytoskeletal proteins and matrix metalloproteinases in dogs with heart failure. Cardiology 110: 230-237. [Crossref]

14. Imai M, Rastogi S, Gupta RC, Mishra S, Sharov VG, et al. (2007) Therapy with cardiac contractility modulation electrical signals improves left ventricular function and remodeling in dogs with chronic heart failure. J Am Coll Cardiol 49: 2120-2128. [Crossref]

15. Morita H, Suzuki G, Haddad W, Mika Y, Tanhehco EJ, et al. (2004) Long-term effects of non-excitatory cardiac contractility modulation electric signals on the progression of heart failure in dogs. Eur J Heart Fail 6: 145-150. [Crossref]

16. Borggrefe MM, Lawo T, Butter C, Schmidinger H, Lunati M, et al. (2008) Randomized, double blind study of non-excitatory, cardiac contractility modulation electrical impulses for symptomatic heart failure. Eur Heart $J$ 29: 1019-1028. [Crossref]

17. Schau T, Seifert M, Meyhöfer J, Neuss M, Butter C (2011) Long-term outcome of cardiac contractility modulation in patients with severe congestive heart failure. Europace 13: 1436-1444. [Crossref]

18. Kadish A, Nademanee K, Volosin K, Krueger S, Neelagaru S, et al. (2011) A randomized controlled trial evaluating the safety and efficacy of cardiac contractility modulation in advanced heart failure. Am Heart J 161: 329-337. [Crossref]

19. Neelagaru SB, Sanchez JE, Lau SK, Greenberg SM, Raval NY, et al. (2006) Nonexcitatory, cardiac contractility modulation electrical impulses: Feasibility study for advanced heart failure in patients with normal QRS duration. Heart Rhythm 3: 1140-1147. [Crossref]

20. Pappone C, Rosanio S, Burkhoff D, Mika Y, Vicedomini G, et al. (2002) Cardiac contractility modulation by electric currents applied during the refractory period in patients with heart failure secondary to ischemic or idiopathic dilated cardiomyopathy Am J Cardiol 90: 1307-1313. [Crossref]

21. Nägele H, Behrens S, Eisermann C (2008) Cardiac contractility modulation in nonresponders to cardiac resynchronization therapy. Europace 10: 1375-1380. [Crossref]

Copyright: $(02015$ Shen E. This is an open-access article distributed under the terms of the Creative Commons Attribution License, which permits unrestricted use, distribution, and reproduction in any medium, provided the original author and source are credited. 